\title{
Rapid Optimization of Working Parameters of Microwave-Driven Multilevel Qubits for Minimal Gate Leakage
}

\author{
Zhongyuan Zhou, ${ }^{1,2}$ Shih-I Chu, ${ }^{1}$ and Siyuan $\operatorname{Han}^{2}$ \\ ${ }^{1}$ Department of Chemistry, University of Kansas, Lawrence, Kansas 66045, USA \\ ${ }^{2}$ Department of Physics and Astronomy, University of Kansas, Lawrence, Kansas 66045, USA
}

(Received 3 February 2005; published 13 September 2005)

\begin{abstract}
We propose an effective method to optimize the working parameters (WPs) of microwave-driven quantum gates implemented with multilevel qubits. We show that by treating transitions between each pair of levels independently, intrinsic gate errors due primarily to population leakage to undesired states can be determined by spectroscopic properties of the qubits and minimized by choosing proper WPs. The validity and efficiency of the approach are demonstrated by applying it to optimize the WPs of two coupled rf SQUID flux qubits for controlled-NOT operation. The result of this independent transition approximation (ITA) is in good agreement with that of dynamic method (DM). The ratio of the speed of ITA to that of DM scales exponentially as $2^{n}$ when the number of qubits $n$ increases.
\end{abstract}

DOI: 10.1103/PhysRevLett.95.120501

A practical quantum computer would be comprised of a large number of coupled qubits which must be kept in highdegree quantum coherence states for a sufficiently long time. During the past decade, significant progress has been made on quantum computation. High-degree quantum coherence has been demonstrated experimentally in systems such as trapped ions [1,2], nuclear spins [3,4], atoms in optical resonators [5], and photons in microwave cavities [6]. However, it seems quite difficult to realize a large number of coupled qubits using these systems. Meanwhile, solid-state qubits are of particular interest because of their advantages of large-scale integration, flexibility in design, and easy connection to conventional electronic circuits [7]. Of those, qubits based on superconducting devices have recently attracted much attention [8] as manipulation of quantum coherent states has been successfully demonstrated in a variety of single qubits [9-13] and coupled two-qubit systems [14-18].

However, the solid-state qubits demonstrated in experiments so far all have relatively short coherence time and high probability of gate errors [9-15,17]. One of the causes of these problems is extrinsic gate error arising from interaction between the environment and qubits resulting in decoherence, such as dephasing and relaxation [8]. Another cause is intrinsic gate error resulting from population leakage to undesired states due to the typical multilevel structures of solid-state qubits $[19,20]$. The intrinsic gate error is crucial since it determines the ultimate performance of the quantum gates and cannot be eliminated by reducing the environmentally caused decoherence.

The leakage of a gate can be characterized quantitatively by summing up the maximum transition probabilities to all undesired states of a multilevel qubit $[19,20]$. It depends strongly on energy level structure and transition matrix elements, i.e., spectroscopic properties of the multilevel qubit determined completely by device parameters (DPs) and external control parameters of the qubit, which we call working parameters (WPs) for simplicity. For instance,
PACS numbers: 03.67.Lx, 85.25.Dq, 89.70.+c

inductance (capacitance) of the superconducting flux (charge) qubit is a DP while external flux (gate voltage) is a WP. For the multilevel qubit with given DPs, the gate leakage is sensitive to their WPs and thus can be minimized by the use of appropriate WPs.

Conventionally, the transition probabilities to all undesired states are calculated by numerically solving the timedependent Schrödinger equation (TDSE) [20,21]. However, this kind of dynamic method (DM) not only needs complicated numerical algorithms, but also a large amount of computational resources. As a result, to optimize the WPs of a many-qubit network for a practical quantum computer using DM may only be possible with ad hoc powerful quantum computers in the future. Thus, a much faster approach is highly desirable.

In this Letter, we propose a very fast method to minimize the leakage of microwave-driven quantum logical gates by choosing appropriate WPs in a system of multilevel qubits with their DPs given in prior. We consider the case of weak microwave fields only since strong fields usually cause many additional types of intrinsic gate errors and thus should be avoided in general [20]. Our method is based on an independent transition approximation (ITA) in which transitions in multilevel qubits are treated independently. The leakage is estimated using the spectroscopic properties and minimized by optimizing the WPs of the qubits. The method is applied to minimize leakage of a controlled-NOT (CNOT) gate implemented with coupled rf superconducting quantum interference device (SQUID) flux qubits. The result is in good agreement with that obtained from DM. More importantly, the ITA is scalable as the number of qubit $n$ increases because the ratio of the speed of ITA to that of DM scales exponentially as $2^{n}$.

In general, for a microwave-driven gate, correlation and interference between transitions of computational states cannot be ignored and transition probability can only be computed accurately by solving TDSE. However, in the case of weak fields considered here, only the transitions 
between levels with which the microwave field is resonant or nearly resonant are significant. For a given level, if the level spacings between it and all other levels are sufficiently different, the correlation and interference between the transitions from this level have negligible effect. Hence, each transition is expected to take place independently and the two levels involved can thus be treated as if they are isolated from the others. For each two-level subsystem interacting with a rectangular pulse, the transition probability can be approximated by an analytical expression using the rotating-wave approximation. Assuming the rectangular pulse is $\epsilon(t)=\epsilon_{0} \cos (\omega t)$, the interaction between the qubit and the pulse is then $V(t)=-\mu \epsilon(t)$, where $\epsilon_{0}$ and $\omega$ are the amplitude and frequency of the pulse and $\mu$ is the dipole moment operator of the qubit. The maximum transition probability from state $i$ to $j$ in an $N$-photon process, $\mathcal{P}_{i j}$, is [22]

$$
\mathcal{P}_{i j}=\Omega_{i j}^{2} /\left(\mathcal{D}_{i j}^{2}+\Omega_{i j}^{2}\right),
$$

where, $\Omega_{i j}=2 \mu_{i j} \epsilon_{0} N J_{N}\left(y_{i j}\right) / y_{i j}$ is the Rabi frequency for the $N$-photon resonance, $\mathcal{D}_{i j}=\Delta E_{i j} / \hbar-N \omega$ is the detuning, $\mu_{i j}=\langle i|\mu| j\rangle$ is the dipole transition matrix element, $\Delta E_{i j}=\left|E_{j}-E_{i}\right|$ is the level spacing between the states $i$ and $j, J_{N}$ is the Bessel function of integer order $N$, and $y_{i j}=\mathbf{d}_{i j} \cdot \epsilon_{0} / \hbar \omega$ with $\mathbf{d}_{i j}=\mu_{j j}-\mu_{i i}$. Note that $\mathcal{P}_{i j}, \Omega_{i j}$, and $\mathcal{D}_{i j}$ depend on the number of photons $N$. In weak fields characterized by $\Omega_{i j} \ll \omega$ only transitions with small $N$ are important. Since the leakage is calculated from the maximum transition probabilities which are independent of pulse shape in weak fields [23] the results obtained here are also valid for other pulse shapes.

For the microwave-driven gate considered here, the microwave is resonant with a pair of computational states. According to Eq. (1), the leakage is suppressed if $\mathcal{D}_{i j} \gg$ $\Omega_{i j}$ holds for all unintended transitions. This condition may be satisfied by using sufficiently weak fields and/or qubits with proper spectroscopic properties. However, the use of exceedingly weak fields will make the gate very slow. Even so, the unintended transitions will still cause large leakage at or near resonance where $\mathcal{D}_{i j} \simeq 0$ and $\mathcal{P}_{i j} \simeq 1$, regardless of intensity of the fields. Thus the leakage can be greatly reduced by setting the level spacings of the qubits substantially detuned from the microwave frequency and/or the dipole transition matrix elements sufficiently small for all unintended transitions.

To investigate the leakage in a multilevel qubit, we study what happens when the microwave acts on each of the computational states, which we call a component of the gate. For an $n$-bit gate, there are $M=2^{n}$ components. The leakage of the $i$ th component is defined as $\eta_{i}=\sum_{k} P_{i k}$, where $P_{i k}$ is the maximum probability of occupying an undesired state $k$ through all possible multiphoton transitions and the sum is over all the undesired states including all noncomputational states as well as those computational states to which no transition is intended. The leakage of the $n$-bit gate, $\eta$, is then defined as $\eta=\max \left(\eta_{1}, \eta_{2}, \cdots \eta_{M}\right)$ which is a function of the $\nu$ WPs $q_{1}, q_{2}, \cdots$, and $q_{\nu}$. The set of WPs, $q_{1}^{\mathrm{opt}}, q_{2}^{\mathrm{opt}}, \cdots$, and $q_{\nu}^{\mathrm{opt}}$ is obtained by minimizing the leakage of gate, namely, $\eta\left(q_{1}^{\mathrm{opt}}, q_{2}^{\mathrm{opt}}, \cdots, q_{\nu}^{\mathrm{opt}}\right) \equiv$ $\min \left[\eta\left(q_{1}, q_{2}, \cdots, q_{\nu}\right)\right]$.

As an example, we apply ITA to optimize the WPs of coupled rf SQUID flux qubits for CNOT gate. Each rf SQUID consists of a superconducting loop of inductance $L$ interrupted by a Josephson junction with critical current $I_{c}$ and shunt capacitance $C$ [24]. A flux-biased rf SQUID with total flux $\Phi$ enclosed in the loop is analogous to a particle of mass $m=C \Phi_{0}^{2}$, where $\Phi_{0}=h / 2 e$ is the flux quantum. The Hamiltonian is $h(x)=p^{2} / 2 m+m \omega_{L C}^{2}(x-$ $\left.x_{e}\right)^{2} / 2-E_{J} \cos (2 \pi x)$. Here, $x=\Phi / \Phi_{0}$ is the canonical coordinate of the particle, $p=-i \hbar \partial / \partial x$ is the canonical momentum conjugate to $x, E_{J}=\hbar I_{c} / 2 e=m \omega_{L C}^{2} \beta_{L} / 4 \pi^{2}$ is the Josephson coupling energy, $\beta_{L}=2 \pi L I_{c} / \Phi_{0}$ is the potential shape parameter, $\omega_{L C}=1 / \sqrt{L C}$ is the characteristic frequency of the SQUID, and $x_{e}=\Phi_{e} / \Phi_{0}$ is the normalized external flux.

The coupled rf SQUID qubits comprise two rf SQUID qubits: a control qubit and a target qubit coupled via their mutual inductance $M[7,25]$. For simplicity, assuming the two SQUIDs are identical: $C_{i}=C, L_{i}=L$, and $I_{c i}=I_{c}$ for $i=1$ and 2. The Hamiltonian of the coupled qubits is $H\left(x_{1}, x_{2}\right)=h\left(x_{1}\right)+h\left(x_{2}\right)+h_{12}\left(x_{1}, x_{2}\right)$, where $h_{12}\left(x_{1}, x_{2}\right)=$ $m \omega_{L C}^{2} \kappa\left(x_{1}-x_{e 1}\right)\left(x_{2}-x_{e 2}\right)$ is the interaction between the qubits, $\kappa=M / L$ is the coupling constant, and $x_{i}, x_{e i}$, and $h\left(x_{i}\right)(i=1$ and 2) are the canonical coordinate, normalized external flux, and Hamiltonian of the $i$ th single qubit. Note that each qubit is a multilevel system. The eigenstate $\mid n)$ and eigenenergy $E_{n}$ of the coupled qubits are computed by numerically solving the eigenvalue equation of $H\left(x_{1}, x_{2}\right)$ using the two-dimensional Fourier-grid Hamiltonian method [26]. They are functions of the WPs $x_{e 1}, x_{e 2}$, and $\kappa$ for given device parameters $L, C$, and $I_{c}[27,28]$. For weak coupling $\kappa \ll 1$, the eigenstate of the coupled qubits, denoted by $|n\rangle=|i j\rangle$, can be well approximated by the product of the control qubit's state $|i\rangle$ and the target qubit's state $|j\rangle, \mid n)=|i\rangle|j\rangle$. When biased at $x_{e 1}, x_{e 2} \approx 1 / 2$ the potential of coupled qubits have four wells $[7,25]$. The lowest eigenstates in each of the four wells, denoted as $|1\rangle=|00\rangle,|2\rangle=|01\rangle,|3\rangle=|10\rangle$, and $|4\rangle=|11\rangle$, are used as the computational states of the coupled qubits. For SQUIDs with $L=100 \mathrm{pH}, C=40 \mathrm{fF}$, and $\beta_{L}=1.2$, the energy levels and level spacings versus $x_{e 2}$ and $\kappa$ are plotted in Figs. 1(a)-1(d), respectively. It is shown that both the energy levels and level spacings are sensitive to the WPs $x_{e 2}$ and $\kappa$. When $x_{e 2}<0.49877$ or $x_{e 2}>0.50123$ in Fig. 1(a) or $\kappa>7.5 \times 10^{-3}$ in Fig. 1(c) the energy level structures become quite complicated. Figs. 1(b) and 1(d) also show that in certain regions of the WP space the level spacings become crowded, i.e., they are degenerate or nearly degenerate, which may result in significant intrinsic gate errors. 

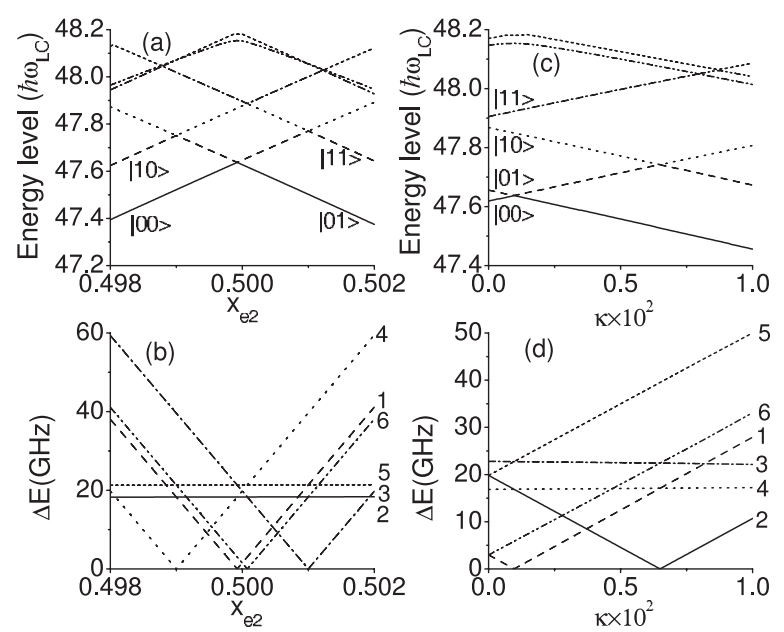

FIG. 1. Energy levels and level spacings of the coupled rf SQUID flux qubits. (a) the energy levels and (b) the level spacings vs $x_{e 2}$ for $x_{e 1}=0.499$ and $\kappa=5 \times 10^{-4}$, (c) the energy levels and (d) the level spacings vs $\kappa$ for $x_{e 1}=0.499$ and $x_{e 2}=$ 0.49985 . The lines $1-6$ in (b) and (d) represent the level spacings $\Delta E_{12}, \Delta E_{13}, \Delta E_{14}, \Delta E_{23}, \Delta E_{24}$, and $\Delta E_{34}$, respectively.

Controlled two-bit gates can be realized by applying a resonant microwave pulse to the target qubit. The interaction between the microwave and the coupled qubits can be written as $V\left(x_{1}, x_{2}, t\right)=m \omega_{L C}^{2}\left[\left(x_{2}-x_{e 2}\right)+\kappa\left(x_{1}-x_{e 1}\right)+\right.$ $\left.x_{m} / 2\right] x_{m}$, where $x_{m}(t)=x_{m 0} \cos (\omega t)$ is the magnetic flux (normalized to $\Phi_{0}$ ) coupled to the target qubit from the microwave with amplitude $x_{m 0}$ and frequency $\omega$. For the CNOT gate, a $\pi$ pulse with $\omega=\Delta E_{34} / \hbar$, where $\Delta E_{34}=$ $\left|E_{4}-E_{3}\right|$, is used. Populations of the states $|10\rangle$ and $|11\rangle$ are exchanged after the $\pi$ pulse only if the initial state is $|10\rangle$ or $|11\rangle$ or a linear combination of them. Note that in this case in addition to the noncomputational states, the undesired states also include the computational states $|00\rangle$ and $|01\rangle$. Using Eq. (1) and following the steps described above, the leakage of the CNOT gate $\eta=\max \left(\eta_{|00\rangle}, \eta_{|01\rangle}\right.$, $\left.\eta_{|10\rangle}, \eta_{|11\rangle}\right)$ is calculated with up to 3 -photon processes included. The $N$-photon processes for $N>3$ have negligible effects. For the SQUID qubits with the previously given DPs, the leakage is a function of WPs $x_{e 1}, x_{e 2}$, and $\kappa$. In Fig. 2(a) we plot the leakage of the CNOT gate versus $x_{e 2}$ and $\kappa$ for $x_{e 1}=0.499$ and $x_{m 0}=2 \times 10^{-4}$. It is shown that the leakage of the CNOT gate is much smaller when the coupled SQUID qubits are operated around $x_{e 2}=0.4997$ and $\kappa=7.5 \times 10^{-4}$ for $x_{e 1}=0.499$.

To evaluate the results obtained from ITA, we performed an accurate dynamic calculation by solving the TDSE of the coupled qubits $i \partial c_{n}(\tau) / \partial \tau=\sum_{n^{\prime}} H_{n n^{\prime}}^{R}(\tau) c_{n^{\prime}}(\tau)$ for the probability amplitudes $c_{n}$ of the first 20 eigenstates $|n|$ using the split-operator method [29], where $\tau=\omega_{L C} t$ and $H_{n n^{\prime}}^{R}=\left[E_{n} \delta_{n n^{\prime}}+\left(n|V| n^{\prime}\right)\right] / \hbar \omega_{L C}[25]$. The probability of being in the state $\mid n)$ is $\left|c_{n}\right|^{2}$, from which the maximum probabilities on the undesired states and the leakage $\eta\left(x_{e 1}\right.$, $\left.x_{e 2}, \kappa\right)$ of the CNOT gate are calculated. The results are

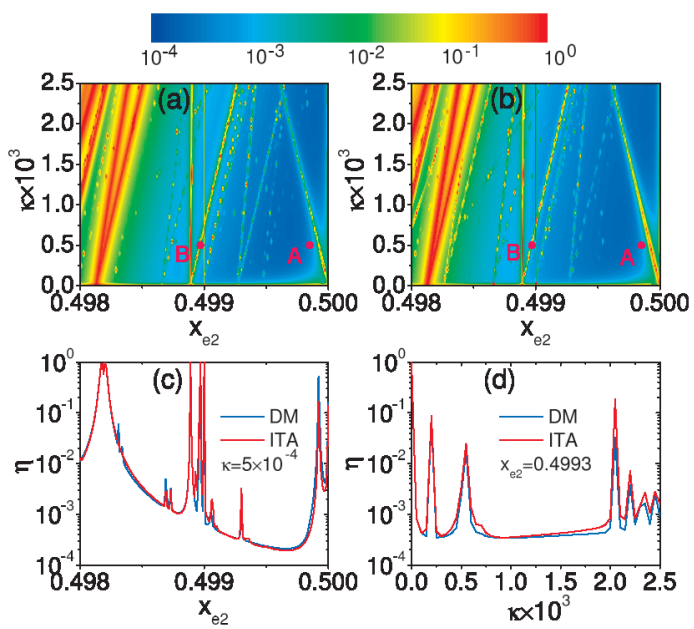

FIG. 2 (color). Leakage of the CNOT gate for $x_{e 1}=0.499$ and $x_{m 0}=2 \times 10^{-4}$. (a) the leakage vs $x_{e 2}$ and $\kappa$ obtained from ITA, (b) the leakage vs $x_{e 2}$ and $\kappa$ obtained from DM, (c) comparison of the leakage vs $x_{e 2}$ at $\kappa=5 \times 10^{-4}$, and (d) comparison of the leakage vs $\kappa$ at $x_{e 2}=0.4993$.

shown in Fig. 2(b) with the color scale identical to Fig. 2(a). For a more quantitative comparison, we also plot, in Figs. 2(c) and 2(d), two line-cut figures at places with the richest structures in Figs. 2(a) and 2(b). The good agreement between the results of ITA and DM demonstrates the validity of ITA. Note that the results of ITA are reliable only for the case of weak fields.

One of the advantages of ITA is that it provides clear physical intuition and insight into the origin of intrinsic gate errors and thus how to reduce the problem by selecting appropriate WPs. Furthermore, ITA is orders of magnitude faster than DM. For instance, the result shown in Fig. 2(a) took less than $3 \mathrm{~h}$ to compute on a dual-2.8 $\mathrm{GHz}$ processor Dell PRECISION 650 workstation, while that shown in Fig. 2(b) took more than $300 \mathrm{~h}$ on the same computer. Denoting $\tau_{S}$ and $\tau_{T}$, respectively, the time needed to calculate the spectroscopic properties of an $n$-bit gate and that used to compute the probability evolution in DM for each component. The total times needed to compute the leakage by ITA and DM are approximately $\tau_{I} \approx \tau_{S}$ and $\tau_{D} \approx \tau_{S}+2^{n} \tau_{T}$, respectively, where the factor $2^{n}$ is the number of components for the $n$-bit gate. Hence, $\tau_{S}<\tau_{T}$ and the ratio $\tau_{D} / \tau_{I} \approx 2^{n} \zeta$ increases exponentially with $n$, where $\zeta \equiv \tau_{T} / \tau_{S}$. For weak fields, $\zeta \gg 1$ and the ratio $\tau_{D} / \tau_{I}$ is very large. For example, for the CNOT gate above $\zeta \sim 25$ and $\tau_{D} / \tau_{I} \sim 100$. Thus as the number of qubits increases the optimization of WPs of multibit quantum gates could become an intractable problem using DM.

To demonstrate quantitatively the effect of different WPs on intrinsic gate errors, we plot in Figs. 3(a) and 3(b) the population evolution of the computational states $|10\rangle$ and $|11\rangle$ for the CNOT gate operated with two different sets of WPs corresponding to points " $A$ " and " $B$ " marked in Fig. 2(a) [also 2(b)], respectively. It is clearly shown that 


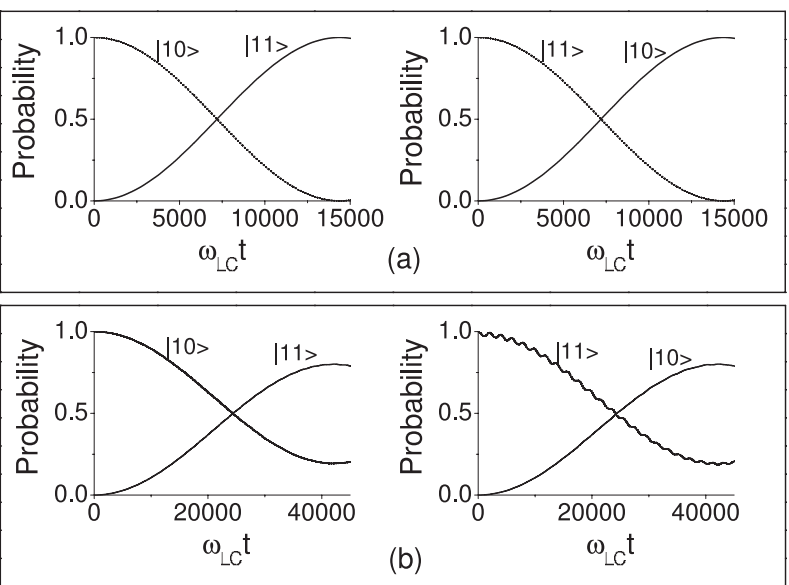

FIG. 3. Population evolution of the computational states $|10\rangle$ and $|11\rangle$ for the CNOT gates with different WPs: (a) $x_{e 2}=$ 0.49985 and $\kappa=5 \times 10^{-4}$ corresponding to the point $\mathrm{A}$ in Fig. 2(a) [also 2(b)], and (b) $x_{e 2}=0.49897$ and $\kappa=5 \times 10^{-4}$ corresponding to the point B in Fig. 2(a) [also 2(b)]. In both cases, $x_{m 0}=2 \times 10^{-4}$ and $x_{e 1}=0.499$.

inversion from the initial state $|10\rangle(|11\rangle)$ to the final state $|11\rangle(|10\rangle)$ after the $\pi$ pulse is almost complete when operated at the point $\mathrm{A}$, but significantly incomplete at the point $\mathrm{B}$. We also computed the population evolution of the coupled qubits with the initial states $|00\rangle$ and $|01\rangle$ using the same microwave pulse. The system essentially stayed in the initial states for the gate operated at the point $\mathrm{A}$, but leaked significantly to the noncomputational states for the gate at the point $\mathrm{B}$. The quality of a gate can be described by gate fidelity $F \equiv \overline{\operatorname{Trace}\left[\rho_{P} \rho_{I}\right]}$, where $\rho_{P}$ and $\rho_{I}$ are the physical and ideal density matrices after gate operation and the overline denotes averaging over all possible initial states [30]. For the CNOT gates operated at the point A we obtain $F_{A}=0.9997$, which is very close to the ideal CNOT gate. In contrast, the gate operated at the point $\mathrm{B}$ has $F_{B}=0.8061$, which is too large to be tolerated. Furthermore, at the point $A$ the gate is about a factor of 3 faster than that at the point $\mathrm{B}$.

In summary, a very efficient method is proposed to optimize the WPs of microwave-driven quantum logical gates in multilevel qubits based on ITA. In this method, the leakage is determined by the spectroscopic properties of the qubits and minimized by choosing proper WPs. This method is exemplified by optimizing the WPs of coupled rf SQUID flux qubits for minimal leakage of the CNOT gate. The result is in good agreement with that of DM. Compared to the conventional DM the ITA not only provides physical insight into the origin of gate leakage but also reduces the computational time significantly. Furthermore, since the ratio of the speed of ITA to that of DM scales exponentially as $2^{n}$, the ITA is scalable as the number of qubit $n$ increases. Our calculation also shows that high intrinsic fidelity CNOT gate can be achieved using microwave-driven rf SQUID qubits with proper WPs. Although the ITA is only applied, as an example, to the rf SQUID qubits, it is also valid for other microwavedriven multilevel qubits. Therefore, ITA provides a much needed solution for the optimization of WPs of multibit gates implemented with multilevel physical qubits, for which DM is extremely time consuming or could even become intractable.

This work was supported in part by the NSF (DMR0325551) and AFOSR, NSA and ARDA through DURINT Grant No. (F49620-01-1-0439).

[1] J. I. Cirac and P. Zoller, Phys. Rev. Lett. 74, 4091 (1995).

[2] C. Monroe et al., Science 272, 1131 (1996).

[3] N.A. Gershenfeld and I. L. Chuang, Science 275, 350 (1997).

[4] J. A. Jones, M. Mosca, and R. H. Hansen, Nature (London) 393, 344 (1998).

[5] Q. A. Turchette et al., Phys. Rev. Lett. 75, 4710 (1995).

[6] M. Brune et al., Phys. Rev. Lett. 77, 4887 (1996).

[7] J. E. Mooij et al., Science 285, 1036 (1999).

[8] Y. Makhlin, G. Schon, and A. Shnirman, Rev. Mod. Phys. 73, 357 (2001).

[9] Y. Nakamura, Y.A. Pashkin, and J.S. Tsai, Nature (London) 398, 786 (1999).

[10] D. Vion et al., Science 296, 886 (2002).

[11] Y. Yu et al., Science 296, 889 (2002).

[12] J. M. Martinis et al., Phys. Rev. Lett. 89, 117901 (2002).

[13] I. Chiorescu et al., Science 299, 1869 (2003).

[14] Y. A. Pashkin et al., Nature (London) 421, 823 (2003).

[15] T. Yamamoto et al., Nature (London) 425, 941 (2003).

[16] A. J. Berkley et al., Science 300, 1548 (2003).

[17] I. Chiorescu et al., Nature (London) 431, 159 (2004).

[18] J. B. Majer et al., Phys. Rev. Lett. 94, 090501 (2005).

[19] R. Fazio, G. M. Palma, and J. Siewert, Phys. Rev. Lett. 83, 5385 (1999).

[20] Z. Zhou, S.-I. Chu, and S. Han, Phys. Rev. B 66, 054527 (2002).

[21] Z. Zhou, S.-I. Chu, and S. Han, Phys. Rev. B 70, 094513 (2004).

[22] M.A. Kmetic, R. A. Thuraisingham, and W. J. Meath, Phys. Rev. A 33, 1688 (1986).

[23] B. W. Shore, The Theory of Coherent Atomic Excitation (John Wiley \& Sons, New York, 1990), Vol. 1.

[24] V. V. Danilov, K. Likharev, and A. B. Zorin, IEEE Trans. Magn. 19, 572 (1983).

[25] Z. Zhou, S.-I. Chu, and S. Han, IEEE Trans. Appl. Supercond. 15, 833 (2005).

[26] S.-I. Chu, Chem. Phys. Lett. 167, 155 (1990).

[27] T. V. Filippov et al., IEEE Trans. Appl. Supercond. 13, 1005 (2003).

[28] A. M. van den Brink and A. J. Berkley, cond-mat/0501148.

[29] M. R. Hermann and J. A. Fleck, Jr., Phys. Rev. A 38, 6000 (1988).

[30] X. Li et al., Science 301, 809 (2003). 